\title{
Alimentación para la actividad física saludable: estudio de caso con escolares
}

\section{Diet for healthy physical activity: a case study with schoolchildren}

1 Rosita Gabriela Flores Robalino

https://orcid.org/0000-0002-4083-585

Universidad Técnica de Ambato, Carrera de Pedagogía de la Actividad Física y Deporte. Tungurahua, Ecuador rg.flores@uta.edu.ec

2 Diana Carolina Acosta Chisaguano iD https://orcid.org/0000-0001-9070-1019 Universidad Técnica de Ambato, Carrera de Pedagogía de la Actividad Física y Deporte. Tungurahua, Ecuador acostadiana123@hotmail.com

3 Gabriela Alexandra Villalba Garzón (iD) https://orcid.org/0000-0001-5589-4927 Universidad Técnica de Ambato, Facultad de Ciencias Humanas y de la Educación, Carrera de Pedagogía de la Actividad Física y Deporte. Ambato, Ecuador ga.villalba@uta.edu.ec

4 Edison Andrés Castro Pantoja $\quad$ (iD) https://orcid.org/0000-0002-4606-5016 Universidad Técnica de Ambato, Carrera de Pedagogía de la Actividad Física y Deporte. Tungurahua, Ecuador ea.castro@uta.edu.ec

Artículo de Investigación Científica y Tecnológica

Enviado: 24/12/2021

Revisado: 29/12/2021

Aceptado: $12 / 01 / 2022$

Publicado:08/03/2023

DOI: https://doi.org/10.33262/concienciadigital.v6i1.4.2024

Flores Robalino, R. G., Acosta Chisaguano, D. C., Villalba Garzón, G. A., \& Castro Pantoja, E. A. (2023). Alimentación para la actividad física saludable: estudio de caso con escolares.

ConcienciaDigital,

6(1.4),

720-733.

https://doi.org/10.33262/concienciadigital.v6i1.4.2024

CONCIENCIA DIGITAL, es una Revista Multidisciplinar, Trimestral, que se publicará en soporte electrónico tiene como misión contribuir a la formación de profesionales competentes con visión humanística y crítica que sean capaces de exponer sus resultados investigativos y científicos en la misma medida que se promueva mediante su intervención cambios positivos en la sociedad. https://concienciadigital.org

La revista es editada por la Editorial Ciencia Digital (Editorial de prestigio registrada en la Cámara Ecuatoriana de Libro con No de Afiliación 663) www.celibro.org.ec 
Palabras claves: nutrientes, hábitos, dieta, escolares.

Keywords: nutrients, habits, diet, school.
Resumen

Introducción. Los hábitos alimenticios en la edad escolar se relacionan directamente con los hábitos de práctica de actividad física, incidiendo en los estilos de vida saludable que estos poseen. Objetivo. Identificar los hábitos alimenticios de los escolares y su relación con los niveles de práctica de actividad física. Metodología. Estudio de enfoque cuantitativo de tipo no experimental, de alcance correlacional con corte transversal, aplicando el cuestionario CHVSAAF para determinación de hábitos alimenticios a una muestra de 84 estudiantes. Resultados. Entre los principales resultados se observa que en la mayoría de los estudiantes existe hábitos alimentarios deficientes, con un nivel medio de práctica de actividad física. Conclusiones. Los datos que se obtuvieron en el estudio nos permitirán buscar alternativas para mejorar los hábitos alimentarios y fomentar la actividad física en los escolares.

\section{Abstract}

Introduction. Eating habits at school age are directly related to the habits of physical activity practice, influencing the healthy lifestyles they have. Objective. To identify the eating habits of schoolchildren and their relationship with the levels of physical activity practice. Methodology. Non-experimental quantitative approach study, of correlational scope with cross-sectional cut, applying the CHVSAAF questionnaire to determine eating habits to a sample of 84 students. Results. Among the main results, it was observed that most of the students had poor eating habits, with an average level of physical activity practice. Conclusions. The data obtained in the study will allow us to look for alternatives to improve eating habits and promote physical activity in schoolchildren.

\section{Introducción}

Los alimentos aportan al organismo el conjunto de bioelementos necesarios para atender las necesidades energéticas (calóricas) y de construcción de nuevas estructuras (plásticas). La mayor parte de los componentes que ingerimos son ab-sorbidos y metabolizados; otros, como la fibra dietética, aunque no son absorbidos o lo son en muy pequeña medida, deben ser aportados necesariamente para garantizar el correcto 
desarrollo de las funciones digestivas. El término alimento es un concepto amplio que se aplica a todo compuesto destinado a la alimentación de los organismos, independientemente de sus características y funciones. Incluye todos los potenciales integrantes de la dieta, sean o no de ingestión obligada, ingeridos por los seres vivos con fines esencialmente nutricionales: crecimiento y desarrollo, aporte calórico, mantenimiento de las funciones biológicas, regulación de la homeostasis (Barbany, 2018).

Se dice que la nutrición es una necesidad orgánica y vital para las personas, mucho más si realiza actividad física. El binomio alimentación - deporte se ve que tiene una estrecha relación con el rendimiento deportivo, equilibrando el gasto energético con la ingesta diaria de alimentos, con ello vamos a disminuir la sensación de agotamiento, si bien en un ámbito eminentemente educativo no tiene demasiada importancia (Lois y Rial, 2016). La mala nutrición en los escolares va a traer consecuencias negativas, por exceso o u desequilibrio dietético que conlleva a cualquier tipo de patología o trastorno. La obesidad es un problema de primer orden en los países industrializados. En la actualidad la obesidad infantil es considerada una epidemia mundial y la OMS lo reconoció en 2002 (Cañizares y Carbonero, 2017).

La educación nutricional es una manera de promover estilos de vida saludables en los escolares, basándose en direccionar diferentes conductas alimentarias adecuadas, aunque también se puede promocionar en otras actividades como el deporte, la actividad física el tiempo para el ocio y las relaciones sociales. Salas-Salvadó et al. (2019) menciona que los hábitos de la alimentación dónde son de naturaleza compleja y susceptible, se refiere a los aspectos familiares, sociales culturales y económicos, además, de las tradiciones y simbolismo en la preparación de los alimentos cuya influencia se presenta por los medios de comunicación y la publicidad (Viera et al., 2018).

La importancia de la alimentación durante la vida escolar es de mantenimiento de la salud para tener un desarrollo adecuado, un mejor crecimiento tanto físico como mental, en la actualidad la calidad nutricional de muchos niños y adolescentes está lejos de llegar alcanzar las recomendaciones de salud. Por ello, es imprescindible que los profesionales en el área, entre los que se incluyen los nutricionistas, puedan detectar las particularidades de cada persona para establecer un programa adecuado de aporte calórico. Acosta (2019), da mayor importancia a la alimentación dando charlas a padres de familia y docentes para adoptar adecuados hábitos alimentarios desde edades tempranas para así prevenir muchas enfermedades en la edad adulta (Esquius de la Zarza y Bach-Faig, 2020).

A muchas personas se les hace difícil poder llevar una vida plena sin la posibilidad de movimiento o interactuar con el mundo, por lo tanto, se puede decir que la nutrición correcta e individualizada es del entrenamiento invisible sin el cual el ejercicio físico no sería posible ni tendría efecto beneficioso que se le atribuye (Lucas, 2021). Debemos 
recalcar que las distintas formas e intensidad en las que cada persona realiza ejercicio físico diario demandarán distintas recomendaciones nutricionales, por lo que es imprescindible que los profesionales en esta área, entre los que se incluyen especialmente los nutricionistas, detectan las particularidades de cada individuo y aconseje las ingestas más adecuadas según el tipo de actividad física desarrollada por cada persona (Esquius de la Zarza y Bach-Faig, 2020).

Si hablamos de actividad física puede ser cualquier movimiento corporal intencional, ejecutado con los músculos esqueléticos, los mismos que están controlados por el cerebro, lo que resulta un gasto de energía y una experiencia personal, esta actividad nos permite interactuar con los seres vivos y el ambiente que nos rodea. Se trata de una definición amplia la que incluye una gran variedad de actividades y de prácticas cotidianas, de trabajo y de ocio de distinta intensidad. Puede incluir actividades poco organizadas como andar, hacer trabajos de jardinería o caseros y otras más organizadas y planificadas y repetitivas que reciben el nombre de ejercicio físico. Incluso debemos aglutinar bajo la definición de actividad física todos los deportes es decir las actividades físicas competitivas reglamentadas e institucionalizadas (Pérez et al., 2021).

De ahí que la actividad física con lleve a ser un factor importante para las personas puesto que debemos tenerla en cuenta cuando hablamos de la salud de los niños y adolescentes. Pero antes de señalar el papel que pueda jugar la actividad física en la salud de la población, querremos sentar las bases de que el lector obtenga una idea clara de qué es la salud y la actividad física, cuáles son las relaciones entre ambos conceptos y qué perspectiva adopta este estudio para considerar que la actividad física y la salud son para todas las personas (Pérez et al., 2021).

La actividad física hace referencia a todo lo que se refiere con movimiento y posturas, la interacción, el cuerpo y la práctica humana (Calderón et al., 2020). Como en muchas otras manifestaciones de la vida, la actividad física forma parte de la dimensión biológica, otra personal y otra sociocultural. De ahí que cualquier intento por explicar y definir que la actividad física debería integrar los tres dimensiones. La forma más extendida de entender la actividad física recoge únicamente la dimensión biológica y se define como cualquier movimiento corporal realizado con los músculos esqueléticos que lleva asociado un gasto de energía (Tigse, 2014). Pero la actividad física también puede ser una de muchas experiencias que vive una persona gracias a la capacidad de los movimientos que la proporciona la naturaleza de cada cuerpo. Estás experiencias nos permiten aprender y valorar pesos y distancias, vivir y apreciar sensaciones muy diversas, adquirir conocimientos de nuestro entorno y nuestro propio cuerpo. Además las actividades físicas forman parte del entorno cultural de la humanidad, desde las más cotidianas cómo andar trotar a otras menos habituales como el fútbol básquet natación o cualquier otro deporte (Pérez at al., 2021). 
Aprender a seleccionar bien los alimentos que consumimos diariamente permite que el organismo puede hacer una buena utilización de sus nutrientes, reparando tejidos generando nuevas células y manteniendo los niveles adecuados en el metabolismo. Esto nos ayuda a prevenir enfermedades y mejorar nuestra vitalidad. Cuando una persona realiza actividad física o deportiva implica un incremento de las necesidades de energía y nutrientes. Este incremento va a variar, puesto que dependerá de la intensidad, la duración y la modalidad de la actividad física, entre otros factores. Aquí la alimentación juega un papel muy importante ya que debe adecuarse para cubrir las necesidades adicionales. Los padres deben tener precaución y evitar que al momento de consumir las comidas se convierten en un medio de castigo, y fomentar que los niños identifiquen las señales fisiológicas del hambre, sed saciedad. De la misma manera, es necesario incentivar a que en los centros educativos aprendan los grupos de alimentos, para que no solo en los hogares sepan llevar una buena alimentación y así saber el rol nutricional que desempeña cada uno de los alimentos o grupos alimentarios (Mena y Cardozo, 2018).

Zapata y Ramírez (2020) quienes citan a varios autores reconocen que la actividad física considerada como una práctica de ejercicios físicos, utilizan a todos los músculos del cuerpo humano, constituyendo un gasto energético que aporta positivamente a la salud especialmente en edades tempranas como la niñez y adolescencia.

La actividad física juega un papel importante en la salud tanto física como mental, y específicamente en la calidad de vida de los niños y jóvenes esto debería ser una prioridad de los Gobiernos. El incluir en el diario vivir la actividad física mejorar no solo la salud sino el bienestar de los niños y adolescentes también representará ahorros considerables en gastos de servicios de salud preventiva, la OMS afirma que la actividad física conduce a un ahorro de 3,2 en gastos médicos. La actividad física de los jóvenes obedece a la ley de la conservación de energía y toda actividad debe extraer energía de los alimentos (Rodríguez, 2019).

En tal sentido la OMS (2010), plantea que los jóvenes en edad escolar deben realizar diariamente al menos 60 minutos cualquier tipo de actividad física de intensidad moderada o vigorosa para garantizar su desarrollo saludable. Con sentido epidemiológico, se afirma que esto puede proporcionarles importantes beneficios físicos, mentales y sociales a los escolares. Por su parte Ayelén (2013), menciona que el realizar la actividad física regular tiene beneficios significativos en la salud de las personas, particular cuando derivan de un plan bien estructurado de ejercicios: el llevar a cabo una actividad que esté planificada, estructurada y que sea repetitiva con el fin de ayudar a mejorar o mantener la actitud física. Diferentes investigaciones sostienen que se debería realizar actividad de moderada intensidad por lo menos 30 minutos diarios. De aquí que se insista sobre la necesidad de generar conciencia en la sociedad, especialmente en la población juvenil, a través de estrategias de prevención, crear las condiciones que hagan segura, posible y 
necesaria la actividad física para que se incorpore como parte de los hábitos saludables en el niño, el adolescente y el adulto, pues la actividad física promueve el mejoramiento de la condición física (Tobar, 2020).

Podemos decir que para ser más precisos en la estimación de los requerimientos energéticos según el grado de actividad física podemos emplear el PAL (Physical Activity Level), que expresa la energía gastada a diario como un múltiplo de la tasa metabólica en reposo:

$$
P A L=\frac{G E T(\text { gasto energético total })}{G M B(\text { gasto energético basal })}
$$

Para estimar los requerimientos energéticos de niños con diferentes niveles de actividad física se resta o se suma un $15 \%$ de la media del PAL $(1,7)$ que refleja un nivel de actividad moderada. Para una actividad ligera $\mathrm{PAL}=1,5$ o intensa $\mathrm{PAL}=2$.

Existe un consenso únicamente en que la realización de actividades deportivas, o simplemente actividades de ejercicio físico conlleva a formar parte de un estilo de vida saludable (Rodríguez et al., 2017). El niño en edad escolar y adolescente debe incluir en su rutina diaria alguna actividad física adecuada largo plazo forme parte de su estilo de vida, el tipo de cantidad de ejercicios no están determinados o limitados aunque se estima que en los escolares y adolescentes se pueda realizar de 30 a 60 minutos de actividad física con una intensidad de moderada a intensa, preferiblemente todos los días a la semana, incentivando la participación de padres e hijos en programas de actividad física bajo una alimentación adecuada, por consiguiente se propone como objetivo identificar los hábitos alimenticios en los escolares con la finalidad de educar y promover hábitos saludables en los escolares.

\section{Metodología}

Para la presente investigación se aplicó el enfoque cuantitativo de tipo básico no experimental, por alcance de tipo correlacional con un corte transversal. Según Sánchez Flores (2019), la investigación bajo el enfoque cuantitativo se denomina así porque trata con fenómenos que se pueden medir a través de la utilización de técnicas estadísticas para el análisis de los datos recogidos, su propósito más importante radica en la descripción, explicación, predicción y control objetivo de sus causas y la predicción de su ocurrencia a partir del desvelamiento de las mismas, fundamentando sus conclusiones sobre el uso riguroso de la métrica o cuantificación, tanto de la recolección de sus resultados como de su procesamiento, análisis e interpretación, a través del método hipotético-deductivo.

La población que intervino en la presente investigación es un total de 84 estudiantes. No requiere la muestra porque la población no supera las 100 personas. 
Se utilizó la técnica de encuesta para los estudiantes, evidenciando como se relaciona la alimentación en la actividad física, lo cual permitió el conocimiento de lo que ocurre en torno al problema planteado en la investigación, dando explicación a las variables de estudio.

La muestra estuvo compuesta por 84 estudiantes con edades comprendidas entre 9 y 12 años de los cuales 38 (45\%) estudiantes son de sexo masculino y 46 (55\%) de sexo femenino.

Para este proyecto de investigación se describirán las variables: La alimentación y la actividad física de los escolares, a partir de esto se podrá obtener gran parte de la información que se recolectará y formará parte del sustento científico.

Para cumplir los protocolos establecidos fue necesario recopilar la información mediante la aplicación del cuestionario de hábitos alimentarios CHVSAAF, el cual es validado estadísticamente en procesos anteriores (Guerrero et al., 2014). El cuestionario consta de 27 ítems, las preguntas 1, 4, 7, 10, 13,16, 19, 22 y 25 miden la dimensión de actividad física, y las preguntas 2, 3, 5, 6, 8, 9, 11, 12, 14,15, 17, 18, 20, 21, 23, 24, 26 y 27 miden la dimensión de alimentación, estructurado con una escala de Likert, cada una con cinco opciones de respuesta: Nunca o menos de 1 vez por mes, 1 a 3 veces por mes, 1 o 2 veces por semana, 3 a 6 veces por semana y diariamente, de la cual se proporcionó información detallada para un adecuado entendimiento de cada uno de los estudiantes. Con la colaboración de los estudiantes se obtuvo la información real, dando validez y veracidad a la investigación.

\section{Resultados}

Como resultados de la investigación se planteó un proceso inicial de caracterización de la muestra de estudio (tabla 1).

Tabla 1

Caracterización de la muestra de estudio

\begin{tabular}{|c|c|c|c|c|c|c|c|c|}
\hline \multirow{2}{*}{ Sexo } & \multirow{2}{*}{$\mathrm{f}$} & \multirow{2}{*}{$\%$} & \multicolumn{3}{|c|}{ Edad (años) } & \multicolumn{3}{|c|}{ Peso (kg) } \\
\hline & & & $\mathrm{M}$ & $\mathrm{DS}$ & $\mathrm{P}$ & $\mathrm{M}$ & DS & $\mathrm{P}$ \\
\hline Masculino & 38 & 45.2 & 9.92 & 0.67 & & 30.55 & 3.24 & \\
\hline Femenino & 46 & 54.8 & 10.76 & 1.06 & $0.169 * *$ & 33.10 & 3.96 & $0.458 * *$ \\
\hline Total & 84 & 100.0 & 10.38 & 0.99 & & 31.94 & 3.85 & \\
\hline
\end{tabular}

Nota. Distribución de frecuencias (f) y porcentajes (\%) de la variable del sexo; valores medios (M) y desviaciones estándares (DS) de las variables de la edad y peso con diferencias significativas en un nivel de $\mathrm{P} \geq 0.05$ entre grupos por sexo

En la tabla 1 se puede apreciar que la muestra de estudio está compuesta por 84 estudiantes de los cuales 38 (45.2\%) estudiantes son de sexo masculino y 46 (54.8\%) de 
sexo femenino. Se observa que en relación a la edad y el peso no existe diferencias significativas entre los grupos de estudio.

En relación a los hábitos de alimentación y nutrición proporcionados por el instrumento se observaron los siguientes resultados (tabla 2).

Tabla 2

Promedios por dimensión de alimentación y nutrición

\begin{tabular}{lcc}
\hline \multicolumn{1}{c}{ Dimensiones de alimentación y nutrición } & Media & $\begin{array}{c}\text { Desviación } \\
\text { estándar }\end{array}$ \\
\hline Tomo refrescos o jugos embotellados & 3.10 & 1.37 \\
Tomo leches saborizadas: chocolate, fresa, etc. & 3.32 & 1.37 \\
Como frutas y verduras & 2.56 & 1.48 \\
Desayuno antes de salir de mi casa & 3.45 & 1.16 \\
Como pizzas o hamburguesas & 3.71 & 1.20 \\
Como panes o pastelitos de paquete & 3.48 & 1.41 \\
Si estoy aburrido o triste me da por comer & 3.24 & 1.28 \\
Hago 5 comidas al día (desayuno, lunch, comida, colación de & 4.37 & 0.90 \\
media tarde, cena) & & \\
Como algunas golosinas o frituras como: chicles, caramelos, & 3.60 & 0.71 \\
papas de bolsa, etc. & 3.36 & 1.36 \\
Cuando tengo sed tomo agua sola & & 1.61 \\
Sigo comiendo, aunque ya no tenga hambre, solo por & 2.79 & 1.03 \\
acabarme lo que tengo en el plato. & 2.94 & 1.39 \\
Como al menos 2 frutas al día & 3.96 & 1.11 \\
Cuando veo la televisión como golosinas o frituras & & 1.35 \\
El lunch que como todos los días lo compro en la escuela o en & 4.23 & 1.22 \\
la calle & & 1.18 \\
Si tengo hambre entre comidas, como una fruta & 3.81 & 1.23 \\
A la hora de comer veo la televisión & 3.58 & \\
Como al menos 2 verduras al día & 4.00 & 3.04 \\
Consumos helados, malteadas, café o chocolate, preparados & & \\
con crema batida, cajeta [manjar] o chispas de chocolate & & \\
\hline
\end{tabular}

De acuerdo con la tabla 2 se puede identificar que casi siempre hacen 5 comidas al día (desayuno, lunch, comida, colación de media tarde, cena) con una media de 4.37, al contrario, casi nunca comen frutas y verduras con una media de 2.56. El análisis de estas dimensiones permitió caracterizar a la muestra de estudio en los diferentes niveles de hábitos alimentarios (tabla 3). 
Tabla 3

Nivel de alimentación en base a las dimensiones de alimentación y nutrición

\begin{tabular}{ccc}
\hline Nivel de hábitos alimentarios & Frecuencia & Porcentaje (\%) \\
\hline Hábitos alimentarios deficientes & 38 & 45.2 \\
Hábitos alimentarios suficientes & 35 & 41.7 \\
Hábitos alimentarios saludables & 11 & 13.1 \\
\hline Total & 84 & 100.0
\end{tabular}

En la tabla 3 en este ítem se determinó que una gran parte de los escolares tienen un nivel de hábitos alimenticios deficientes con una frecuencia de 38(45.2\%). Y el nivel de hábitos saludables presenta una frecuencia de 11 (45.1\%).

Se puede decir que la alimentación saludable es aquella que proporciona los nutrientes que el cuerpo necesita para mantener el buen funcionamiento del organismo, conservar o restablecer la salud, de esta manera minimizar el riesgo de enfermedades, garantizar desarrollo y crecimiento adecuado de los escolares.

En relación a los hábitos de actividad física se observaron los siguientes resultados (tabla $4)$.

\section{Tabla 4}

Promedios por dimensión de actividad e inactividad física

\begin{tabular}{lcc}
\hline \multicolumn{1}{c}{ Dimensiones de actividad e inactividad física } & Media & $\begin{array}{c}\text { Desviación } \\
\text { estándar }\end{array}$ \\
\hline $\begin{array}{l}\text { Hago actividades físicas y/o deportivas con mi } \\
\text { familia }\end{array}$ & 3.20 & 1.06 \\
$\begin{array}{l}\text { Estoy más tiempo en la computadora o videojuegos } \\
\text { que jugando fútbol u otro deporte o actividad física } \\
\text { Me paso } 2 \text { horas o más viendo programas de }\end{array}$ & 3.04 & 1.38 \\
$\begin{array}{l}\text { televisión } \\
\text { Juego en el parque, jardín o patio con otros niños }\end{array}$ & 3.65 & 1.32 \\
$\begin{array}{l}\text { A la hora de recreo hago algún deporte o actividad } \\
\text { física }\end{array}$ & 4.01 & 1.16 \\
$\begin{array}{l}\text { Practico algún tipo de actividad física o deportiva } \\
\text { además de la que realizo en la escuela los días de }\end{array}$ & 4.29 & 0.99 \\
$\begin{array}{l}\text { Educación Física } \\
\text { Camino por lo menos 15 minutos por día } \\
\begin{array}{l}\text { Me paso una buena parte de la mañana o tarde } \\
\text { acostado }\end{array}\end{array}$ & 3.99 & 1.25 \\
$\begin{array}{l}\text { Me quedo en casa sentado o acostado, en vez de jugar } \\
\text { o hacer deporte o actividad física }\end{array}$ & 3.52 & 1.26 \\
\hline
\end{tabular}


En la tabla 4 en base a los valores obtenidos podemos determinar qué el valor positivo es a la hora de recreo hago algún deporte o actividad física con una media de 4.63, y el valor negativo es el juego en el parque, jardín o patio con otros niños con una media de 3.0. en base a estas respuestas se establecieron los diferentes niveles de hábitos de práctica de actividad física (tabla 5).

\section{Tabla 5}

Nivel de práctica de actividad física

\begin{tabular}{ccc}
\hline $\begin{array}{c}\text { Niveles de práctica de } \\
\text { actividad física }\end{array}$ & Frecuencia & Porcentaje (\%) \\
\hline Alto & 29 & 34.5 \\
Medio & 31 & 36.9 \\
Bajo & 24 & 28.6 \\
\hline Total & 84 & 100.0 \\
\hline
\end{tabular}

En la tabla 5 se determinó que la mayoría tiene un nivel medio en la práctica de actividad física, con una frecuencia de 31(36.9\%).

Tomando en cuenta que la alimentación es uno de los factores fundamentales para poder realizar ejercicio físico, ya que supone una acumulación de energía, necesaria para el movimiento y, por otro lado, la práctica regular de actividad física condiciona todos los aspectos cualitativos y cuantitativos de la nutrición; por tanto, es una relación bidireccional que en el presente estudio se pudo observar (tabla 6).

\section{Tabla 6}

Relación entre los niveles de hábitos alimentarios y de práctica de actividad física

\begin{tabular}{lcccc}
\hline \multirow{2}{*}{ Nivel de hábitos alimentarios } & \multicolumn{3}{c}{ Nivel de práctica de actividad física } & \multirow{2}{*}{ Total } \\
\cline { 2 - 4 } & Alto & Medio & Bajo & \\
\hline $\begin{array}{l}\text { Hábitos alimentarios } \\
\text { deficientes }\end{array}$ & 1 & 18 & 19 & 38 \\
$\begin{array}{l}\text { Hábitos alimentarios } \\
\text { suficientes }\end{array}$ & 18 & 12 & 5 & 35 \\
$\begin{array}{l}\text { Hábitos alimentarios } \\
\text { saludables }\end{array}$ & 10 & 1 & 0 & 11 \\
\hline Total & 29 & 31 & 24 & 84 \\
\hline
\end{tabular}

Con relación a los hábitos alimentarios deficientes presentan el $45.2 \%$, del cual $50 \%$ representa un nivel bajo de práctica de actividad física, $47.4 \%$ un nivel medio, y el $2.6 \%$ un nivel alto, evidenciando una predominancia en el nivel medio. 
Para determinar estadísticamente la existencia o no de una correlación entre los hábitos alimentarios y de práctica de la actividad física se aplicó la prueba Chi-cuadrado de Pearson (tabla 7).

\section{Tabla 7}

Comprobación estadística de la relación entre los niveles de hábitos alimentarios y de práctica de actividad física

\begin{tabular}{cccc}
\hline Estadísticos & Valor & df & $\begin{array}{c}\text { Significación } \\
\text { asintótica } \\
\text { (bilateral) }\end{array}$ \\
\hline Chi-cuadrado de Pearson & 39.471 & 4 & $0.000^{*}$ \\
\hline N de casos válidos & & \multicolumn{2}{c}{84} \\
\hline
\end{tabular}

Nota. Significación estadística en un nivel de $\mathrm{P}<0.05$ (*)

La prueba estadística utilizada para determinar la relación entre las variables de estudio se baso en un valor límite de aceptación de la hipótesis nula de 0.297 , en base al $99 \%$ (0.01) de confianza y con 4 grados de libertad, el cual es inferior al valor obtenido para la muestra estudiada (39.471), respaldado por un nivel de significación de $\mathrm{P}<0.05$, que afirman la existencia de una relación directa entre el nivel de habitos alimentarios o de alimentación y el nivel de práctica de la actividad física valorada en el estudio.

\section{Conclusiones}

- En la encuesta realizada del nivel de hábitos alimentarios podemos observar como resultado que el $45.2 \%$ los estudiantes tienen hábitos alimentarios deficiente ósea no tienen idea de cómo debería ser una correcta alimentación, siendo si $41.7 \%$ hábitos suficientes y el $13.1 \%$ los estudiantes presentan hábitos saludables.

- Las personas deben considerar la importancia de mantener un estilo de vida activo y por tanto considerar que la actividad física es muy importante para el cuerpo, en los datos analizados de los niveles de práctica de la actividad física podemos observar el $36.9 \%$ que sería un nivel medio de practica de actividad física por parte de los estudiantes, un $34.5 \%$ en un nivel alto y un $28.6 \%$ que equivale a un 24 en un nivel bajo de práctica de actividad física por parte de los estudiantes. En las dimensiones de la actividad física e inactividad física se obtuvo los valores positivos con una media de 4.63 qué es "a la hora de recreo hago algo algún deporte o actividad física", y con una media de 4.29 "practico algún tipo de actividad física o deportiva además de la que realizo en la escuela los días de Educación Física" y asimismo tenemos los valores negativos que en este caso tenemos "estoy más tiempo en la computadora o videojuegos que jugando fútbol y otro deporte o actividad física" que nos da una media de 3.04.

- La alimentación es uno de los factores muy importantes que hay que tomar en cuenta en conjunto cuando nosotros vamos a realizar cualquier tipo de actividad 
física, por lo que el rendimiento deportivo mejorará con una alimentación adecuada, en base a estos criterios en el presente estudio se determinó que existe una relación directa entre el nivel de hábitos alimentarios y de practica de actividad física, lo cual fue analizado y verificado estadísticamente.

\section{Referencias bibliográficas}

Acosta, W. E. C., Hinojosa, J. L. A., Cañizares, C. M. S., \& Bonilla, J. A. M. (2019). Aporte calórico nutricional en la tonificación muscular: Caso de estudio deportistas semiprofesionales de fútbol. Ciencia Digital, 3(2.5), 134-149.

Ayelén, M. (2013). Estudio del estado nutricional, actividad física y alimentación de escolares del nivel primario. Universidad Nacional de la Plata. pg. 35-60

Bach-Faig, A., \& Esquius de la Zarza, L. (2019). La alimentación en la actividad física y el deporte (E. UOC (ed.)).pg. 1-64

Barbany, J. R. (2018). Alimentación para el deporte y la salud. Barcelona, Editorial Paidotribo.pg. 1-32

Calderón, C. F., Neira, G. E., \& Flórez-Méndez, J. (2020). Evaluación de hábitos de alimentación, actividad física y estado nutricional en estudiantes de 4 a 13 años de la comuna de Llanquihue, Chile. Revista Chilena de Nutrición, 47(4). https://doi.org/10.4067/S0717-75182020000400612 pg. 612-619

Cañizares Marquéz, J. M., \& Carbonero Celis, C. (2017). Enciclopedia para Padres, sobre Actividad Física, Salud y Educación en los niños (Wanceulen Editorial (ed.)).

Esquius de la Zarza, L., \& Bach-Faig, A. (2020). Personalización y nuevas tendencias en alimentación deportiva (E. UOC (ed.)). pg. 1-212

Guerrero, G., López, J., Villaseñor, N., Gutiérrez, C., Sánchez, Y., Santiago, L., Martínez, O., \& Maya Lozano, N. (2014). Diseño y validación de un cuestionario de hábitos de vida de alimentación y actividad física para escolares de 8-12 años. Revista Chilena de Salud Pública, 18(3). https://doi.org/10.5354/0719-5281.2014.33915 pg. 249

Lois Carro, L., \& Rial Rebullido, T. (2016). Hábitos alimentarios y de actividad física de alumnado de Educación Primaria: estudio descriptivo de un colegio de Pontevedra. Sportis. Revista Técnico-Científica Del Deporte Escolar, Educación Física y Deporte

Lucas, P. J. C. (2021). The influence of a healthy lifestyle on body mass index in a sample 
of 12-14 year old individuals in Murcia (Spain). Anales Del Sistema Sanitario de Navarra, 44(1). https://doi.org/10.23938/ASSN.0883 pg. 33-40

Mena Bejarano, B., \& Cardozo Vásquez, Y. S. (2018). Actividad física en niños, niñas y adolescentes: Investigación, teoría y práctica (U. N. de Colombia (ed.)).

OMS. (2010). Recomendaciones mundiales sobre actividad física para la salud (O. M. de la Salud (ed.)). pg.100

Pérez Samaniego, V. Peiró Velert, C. \& Devís Devís, J. (Coord.) (2021). Actividad física, deporte y salud. Editorial INDE. .pg.1-20

Rodríguez Escobar, G. (2019). Alimentación y nutrición aplicada (U. del Bosque (ed.)).pg. 84

Rodríguez, M. de la F., Morell, J. M., \& Fresneda, J. (2017). Cuida de mí: Claves de la resiliencia familiar (E. UNED (ed.)).pg.1-70

Salas-Salvadó, J., Bonada, I., Sanjaume, A., Trallero Casañas, R., Saló i Solà, M. E., \& Burgos Peláez, R. (2019). Nutrición y dietética clínica (E. H. Sciences (ed.); 4th ed.).pg. 183

Tigse Osorio, J. A. (2014). La alimentación y su influencia en el desarrollo de la actividad física de los estudiantes de sexto y séptimo año de egb de la escuela Agustín Albán, del cantón Pujilí provincia de Cotopaxi [Universidad Técnica De Ambato]. In Repo.Uta.Edu.Ec.

http://repo.uta.edu.ec/bitstream/handle/123456789/5301/Mg.DCEv.Ed.1859.pdf?se quence $=3$ pg. $1-60$

Tobar, K. D. L., Bonilla, J. A. M., \& Acosta, W. C. (2020). Análisis de la frecuencia cardíaca: un estudio con estudiantes universitarios que practican actividad física regular. Ciencia Digital, 4(1.1.), 21-31.

Viera, E. C., Quiñones, I. T., \& García Araujo, J. A. (2018). Relationship between physical activity, nutrition, and family in school-aged youth. Retos, 2041(34), pg.85-88.

Zapata Galarza, M. V., \& Ramírez Ponce, D. Y. (2020). La promoción de la actividad física para disminuir el sobrepeso en niños. Podium. Revista de Ciencia y Tecnología En La Cultura Física, 15(1), pg. 153-165.

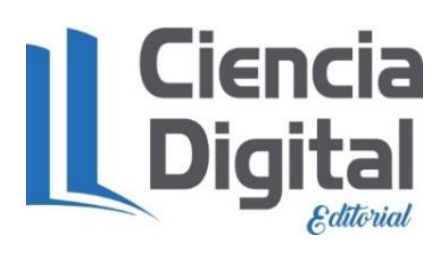


El artículo que se publica es de exclusiva responsabilidad de los autores y no necesariamente reflejan el pensamiento de la Revista Conciencia Digital.

\section{Ciencia}

El artículo queda en propiedad de la revista y, por tanto, su publicación parcial y/o total en otro medio tiene que ser autorizado por el director de la Revista Conciencia Digital.
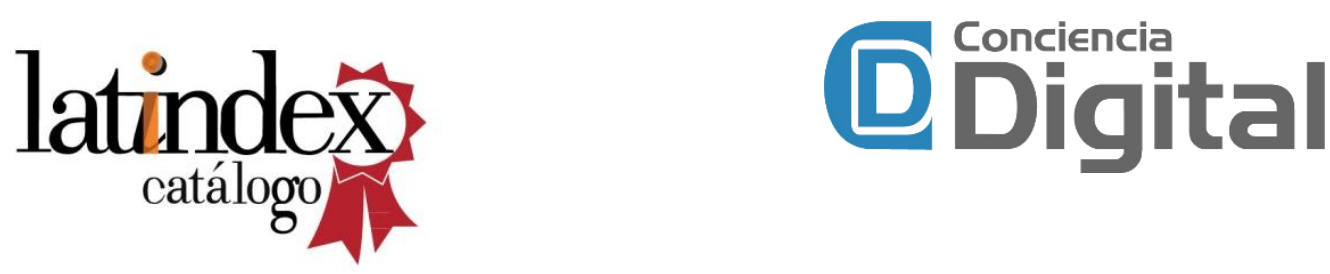

Indexaciones

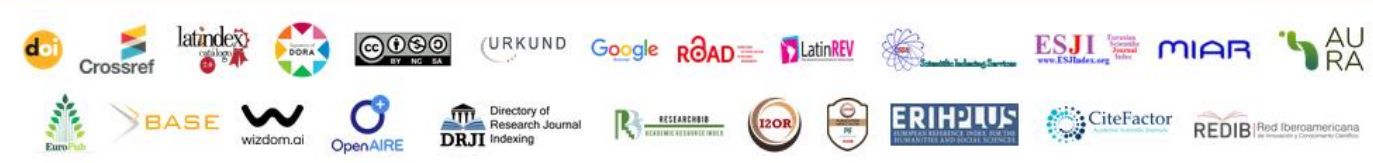

